\title{
Neutrino Super Beam for lepton CP violation discovery based on the European Spallation Source
}

\author{
Eric Baussan* \\ IPHC, Université de Strasbourg, CNRS/IN2P3, F-67037 Strasbourg, France \\ E-mail: eric.baussaneiphc.cnrs.fr
}

on behalf of the ESS $v$ SB Project

\begin{abstract}
For the lepton $\mathrm{CP}$ violation discovery very intense neutrino beams are needed produced using very powerful proton beams. The proposed project, ESSvSB, is based on the European Spallation Source proton linac, the construction has started in 2014 and will finish by 2023. This linac will have a power of $5 \mathrm{MW}$ producing protons of $2 \mathrm{GeV}$ energy. The combination of the high beam intensity and the comparatively low proton energy allows the neutrino measurements to be made with a megaton Water Cherenkov neutrino detector installed $1000 \mathrm{~m}$ down in a mine at $540 \mathrm{~km}$ from the neutrino source, near the position of the second neutrino oscillation maximum. The relative variation of the electron neutrino yield with the $\mathrm{CP}$ violation angle $\delta_{C P}$ is about three times larger than the first maximum. This implies that the measurement of $\delta_{C P}$ is about three times less sensitive to the experimental systematic errors, which is the error determining the ultimate performance for discovery and measurement of leptonic CP violation. This observation has the potential to shed light on the matter-antimatter asymmetry in the Universe. The use of a large underground neutrino detector to measure the proton lifetime, detect cosmological neutrinos and neutrinos from supernova will also be possible.
\end{abstract}

The European Physical Society Conference on High Energy Physics

22-29 July 2015

Vienna, Austria

${ }^{*}$ Speaker. 


\section{Measuring the lepton $\mathrm{CP}$ phase with the second oscillation maximum}

The measurement of the last mixing angle $\theta_{13}$ by the reactor experiments[1, 4] in 2012 has considerably improved our view of the mixing mechanism in neutrino oscillations and has opened the way to the precision era in this field. One of the next challenge will focus on CP violation search in the leptonic sector which will require new facilities with intense neutrino beams. Although neutrino factory remains the ultimate facility to achieve the best sensitivity of the $\delta_{C P}$ phase, the large value of the mixing angle $\theta_{13}$ allows to consider neutrino superbeams as a cost effective option able to give a solution in the next decades. The international community is considering those facilities with several baselines looking at the first oscillation maximum of the $v_{\mu} \rightarrow v_{e}$ oscillation probability. But an interesting option consists at working at the second maximum in which the sensitivity $\delta_{C P}$ is enhanced. The oscillation probability can be expanded in a sum of three terms respectively referenced as atmospheric, solar and an interference term containing the $\mathrm{CP}$ phase:

$$
\begin{aligned}
P_{\substack{v_{\mu} \rightarrow v_{e} \\
P_{e}}} & s_{23}^{2} \cdot \sin ^{2} 2 \theta_{13} \cdot \sin ^{2}\left(\frac{\Delta_{13} L}{2}\right)+c_{23}^{2} \cdot \sin ^{2} 2 \theta_{12} \cdot \sin ^{2}\left(\frac{\Delta_{21} L}{2}\right) \\
& +\widetilde{J} \cdot \cos \left( \pm \delta_{C P}-\frac{\Delta_{13} L}{2}\right) \cdot \sin \left(\frac{\Delta_{21} L}{2}\right) \cdot \sin \left(\frac{\Delta_{31} L}{2}\right)
\end{aligned}
$$

The upper/lower sign refers to neutrinos/antineutrinos, $E_{V}$ being the neutrino energy, $\mathrm{L}$ the sourcedetector distance, $\Delta_{i j}=\frac{\Delta m_{i j}^{2}}{2 E_{v}}$ and $\widetilde{J}=c_{13} \cdot \sin 2 \theta_{12} \cdot \sin 2 \theta_{23} \cdot \sin 2 \theta_{13}$. The differents contributions of these terms are compared in Figure 1 [5] for a small and a large value of $\theta_{13}$. It can be seen that the relative contribution of the interference term, in which the $\delta_{C P}$ phase appears, has a contribution enhanced in the second maximum corresponding to $\mathrm{L} / \mathrm{E}=1500 \mathrm{~km} / \mathrm{GeV}$.
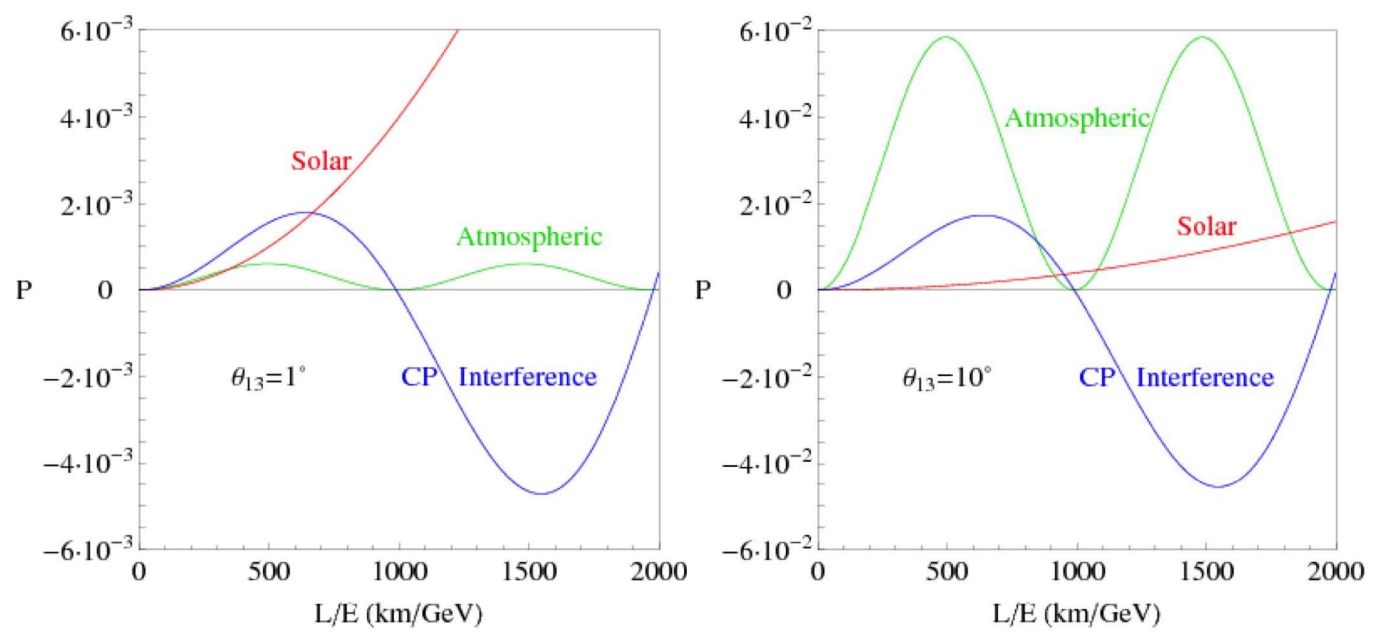

Figure 1: Oscillation probability $v_{\mu} \rightarrow v_{e}$ for small $\left(1^{\circ}\right)$ and large value of $\left(10^{\circ}\right)$ of $\theta_{13}$.

Several research programs in the world are investigating the best facilities to address these questions: Deep Underground Neutrino Experiment[6] (DUNE) in USA, Hyper-K[7] in Japan and ESS $v$ SB in Europe[8]. The ESSvSB collaboration proposes a cost effective solution by using the European Spallation Source currently under construction at Lund in Sweden to produce an 

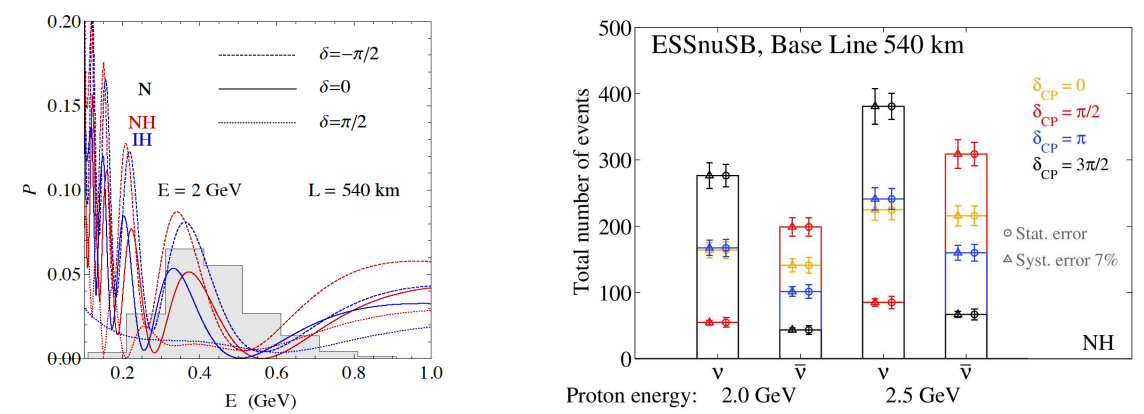

Figure 2: Neutrino energy spectrum for a baseline of $540 \mathrm{~km}$ with $2.0 \mathrm{GeV}$ (Left). Number of $v_{e} / \overline{\nu_{e}}$ events by considering (2+8) years of running for two proton energies of $2.0 \mathrm{GeV}$ and $2.5 \mathrm{GeV}$ (Right).

intense neutrino beam. A Water Cherenkov detector is chosen as far detector and will be located in the Garpenberg mine located at $540 \mathrm{~km}$ from the source. The baseline is then focused on the second oscillation as shown in Figure 2. The ratio between the number of electron neutrinos for $\delta_{C P}=3 \pi / 2$ and $\delta_{C P}=\pi / 2$ reaches 4.8 and appears to be higher by a factor of at least 2.5 compared to experiments operating at the first oscillation maximum[9].

\section{A very intense neutrino super beam with a high $\mathrm{CP}$ violation discovery potential}

The future facilities for precision measurements will require the elaboration of a proton driver at Mega Watt (MW) scale. The European Spallation Source[10] will be able to deliver a 5 MW beam power for neutron experiments in a near future. A modification of the linac is proposed in the ESS $v$ SB project in which the initial mean power will be increased to reach $10 \mathrm{MW}$. The proton driver will be shared simultaneously by neutron (5MW) and neutrino (5MW) applications with no reduction in the spallation neutron prodution. Specific beam profile on the proton pulses is required for neutrino users and imply to adapt the linac parameters.

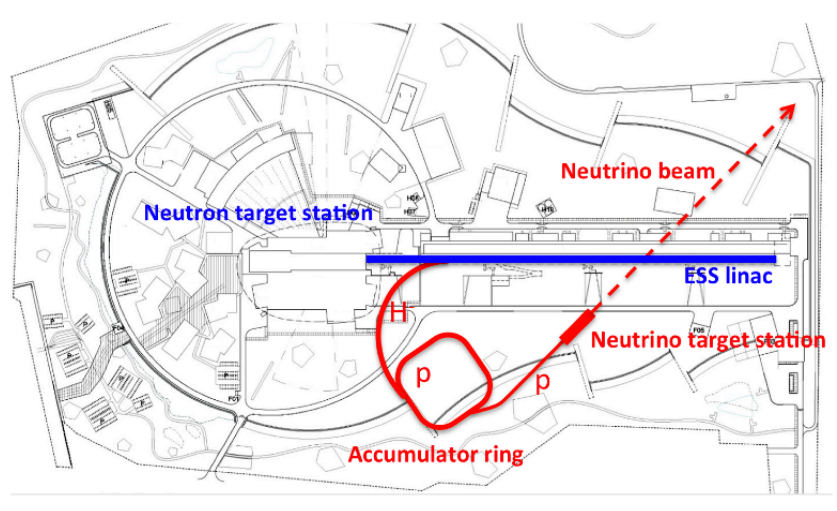

Figure 3: ESS neutrino beam layout.

A accumulator system[11], placed at the end of the linac as illustrated in Fig 3. is essential to reduce the initial proton pulse length from $2.86 \mathrm{~ms}$ to a few $\mu \mathrm{s}$ in order to minimize the duration 
of the current pulses in the beam focusing system. Due to charge space effect at the entrance of the accumulator an $\mathrm{H}^{-}$source has to be implemented in complement with the $\mathrm{H}^{+}$one for neutron mode.

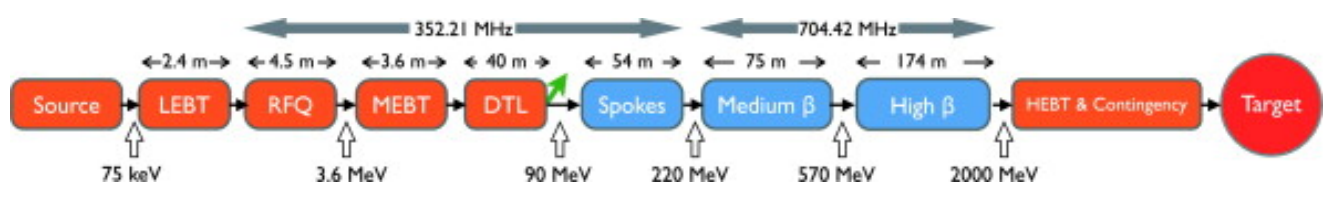

Figure 4: ESS linac implementation.

At the end of the accumulator, the $5 \mathrm{MW}$ proton beam is distributed via four beam-lines onto the four targets of the hadronic collector. The beam is splitted by kicker magnets, bended by dipoles and finally is focused onto the four targets by a system of quadrupoles[12]. Several configurations are under investigation including beam dumps for safety reasons in case of beam defocussing as shown on Figure 5.
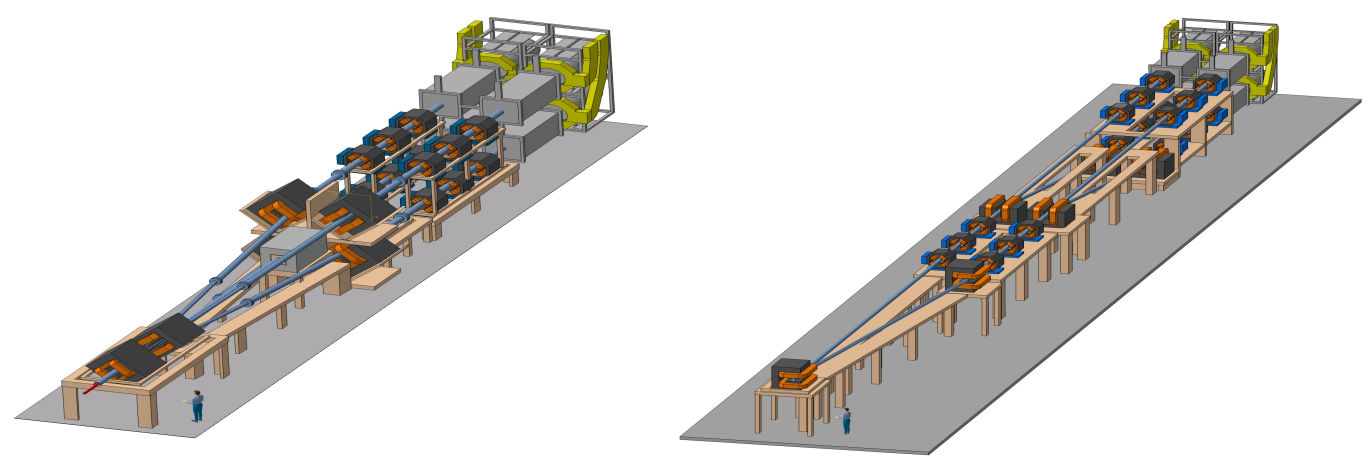

Figure 5: Switchyard option possibilites at the end of the accumulator.

Each target is embedded in a magnetic horn which focus the secondary particles escaping the target into the decay tunnel. A large part of the energy carried out by the proton beam will be released in the surrounding environment of the hadronic collector. A target station concept inspired from EUROnu Design Study[13] with an appropriate shielding has to be implemented in order to be in agreement with the regulation and safety rules.

\section{A hadronic collector design for a proton driver at MW scale}

Compared to the existing neutrino beams, the hadronic collector will be designed to work with $5 \mathrm{MW}$ proton beam power from the ESS linac with a $28 \mathrm{~Hz}$ repetition rate. Many constraints appears du to the high particle rate limiting the lifetime of the whole system. The design proposed by the ESS vSB project is inspired from previous studies[13] and should be able to work under these extreme working conditions. Each proposed element has been studied to take into account the present technological limitations and to provide good reliability during the running time of the experiment: 
- The target technology is based on a packed bed titanium spheres cooled with pressurized helium gas for an efficient cooling.

- For the four horn system shown in Fig 6, a peak current of $350 \mathrm{kA}$ is pulsed at $14 \mathrm{~Hz}$ repetition frequency. About $52 \mathrm{~kW}$ are dissipated from resistive heating in the horn structure. In case of one horn is damaged, the beam power will be shared by the three other horns. Each horn will be able to work under a $1.6 \mathrm{MW}$ beam power.
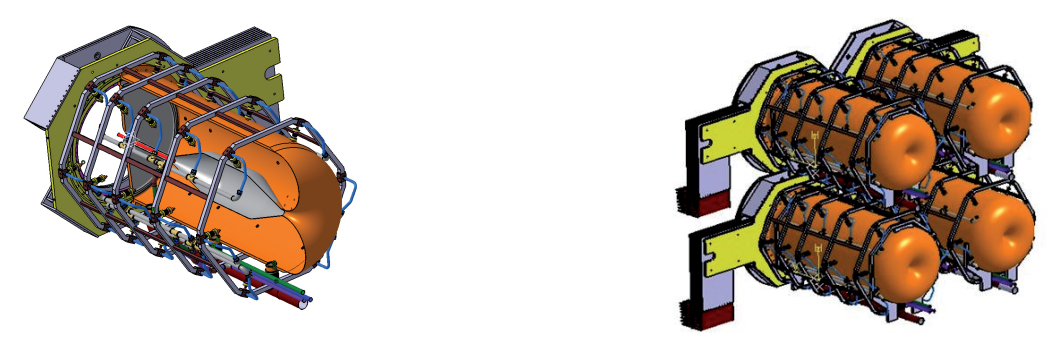

Figure 6: Horn view and four horn system.

- Horn structure: the aluminium skin is made of Al 6061 T6 alloy; good trade-off between mechanical strength, resistance to corrosion and electrical conductivity. The horn skin has to be as thin as possible for the best physics performance and to limit the energy deposition from secondary particles. The horn wall has also to be thick enough to sustain dynamic stress from the current pulses.

- Mechanical studies: a finite element model allows the calculation of the horn stress and deformation due to the magnetic pressure and thermal dilatation minimum stress ( $<30 \mathrm{MPa})$, with a maximal lifetime when the horn has a uniform temperature (around $60^{\circ} \mathrm{C}$ ) maintained by water cooling.

- Heat transfer and cooling: the current is transmitted to each horn by a $33 \mathrm{~m}$ transmition line based on eight aluminum striplines spaced by $1 \mathrm{~cm}$. The dimensions of the striplines allow them to have small resistivity and inductance.

\section{Detector Designs}

The combined experiment of the Super-Beam with MEMPHYS detector[15] has a very good discovery potential of $\mathrm{CP}$ violation and a very reach astrophysics program. MEMPHYS is a proposed 0.5 Mton scale underground Water Cherenkov experiment to be performed deep underground. It consists of two modules of $103 \mathrm{~m}$ height and $65 \mathrm{~m}$ diameter: each module has 120000 8" (or 10") PMTs and 30\% optical coverage. MEMPHYS is considered as the Far Detector (FD) of the ESS $v$ SB facility and will be placed at the Garpenberg Mine at $540 \mathrm{~km}$ from the ESS facility. A Near Detector is necessary to reach the required experimental sensitivity, in particular, to measure the flux, the neutrino cross-sections and to determine the flavor contaminations of the beam to 
reduce the systematics. The ND concept under consideration is also based on Water Cherenkov detector technology which should be able to distinguish charged current interaction and should have good energy reconstruction capability.

\section{Conclusion}

Several options are proposed by the international community. In complement of the lepton CP violating phase $\delta_{C P}$ reseach, the ESS $v$ SB facility offers a large physics program related to nucleon decay, neutrinos from supernovae, solar and atmospheric neutrinos. The ESS $v$ SB project remains the only project in Europe with the most powefull proton driver which should be ready by 2023 horizon.

\section{References}

[1] K. Abe et al. [T2K Collaboration], Phys. Rev. Lett. 107, 041801 (2011) [arXiv:1106.2822 [hep-ex]].

[2] Y. Abe et al. [Double Chooz Collaboration], Phys. Rev. Lett. 108, 131801 (2012) [arXiv:1112.6353 [hep-ex]].

[3] J. K. Ahn et al. [RENO Collaboration], Phys. Rev. Lett. 108, 191802 (2012) [arXiv:1204.0626 [hep-ex]].

[4] F. P. An et al. [Daya Bay Collaboration], Phys. Rev. Lett. 108, 171803 (2012) [arXiv:1203.1669 [hep-ex]].

[5] P. Coloma and E. Fernandez-Martinez, JHEP 1204 (2012) 089 [arXiv:1110.4583].

[6] [DUNE Collaboration], An Experimental Program in Neutrino Physics, Nucleon Decay, and Astroparticle Physics Enabled by the Fermilab Long-Baseline Neutrino Facility, Letter of Intent Submitted to the Fermilab PAC, P- 1062, January 5, 2015, http://www.fnal.gov/directorate/program-planning/Jan2015Public/LOI-LBNF.pdf, https://dune.bnl.gov/tmp/volume-project.pdf

[7] K. Abe et al. [Hyper-Kamiokande Proto- Collaboration], PTEP 2015, no. 5, $053 \mathrm{C} 02$ (2015) [arXiv:1502.05199 [hep-ex]].

[8] E. Baussan et al. [ESSnuSB Collaboration], Phys. Lett. B 885, 127 (2014) [arXiv:1309.7022].

[9] E. Wildner, M. Martini, H. Schonauer, A. Burgman, J. Cederkall, P. Christiansen, T. Ekelof and M. Olvegard, arXiv:1510.00493 [physics.ins-det].

[10] European Spallation Source ESS AB, http://www.esss.se

[11] E. Wildner, J. Jonnerby, J. P. Koutchouk, M. Martini, H. Schönauer, E. Bouquerel, M. Dracos and N. Vassilopoulos et al., Proceedings of IPAC2014, Dresden, Germany

[12] E. Bouquerel, IPAC-2015-MOPWA017.

[13] T. R. Edgecock, O. Caretta, T. Davenne, C. Densham, M. Fitton, D. Kelliher, P. Loveridge and S. Machida et al., Phys. Rev. ST Accel. Beams 16 (2013) 021002 [arXiv:1305.4067].

[14] P. Coloma, P. Huber, J. Kopp and W. Winter, Phys. Rev. D 87 (2013) 3, 033004 [arXiv:1209.5973].

[15] L. Agostino et al. [MEMPHYS Collaboration], JCAP 1301 (2013) 024 [arXiv:1206.6665]. 